\title{
INSUFICIENCIA MESENTÉRICA CRÓNICA LUEGO DE RADIOTERAPIA PARA AÓRTICA*
}

\author{
Drs. Francisco Vargas S. ${ }^{1}$, Renato Mertens M. ${ }^{1}$, Michel Bergoeing R. ${ }^{1}$, \\ Albrecht Krämer S. ${ }^{1}$, Leopoldo Mariné M. ${ }^{1}$, Francisco Valdés E. ${ }^{1}$ \\ 1 Departamento de Cirugía Vascular y Endovascular, Pontificia Universidad Católica de Chile. \\ Santiago, Chile.
}

\begin{abstract}
\section{Surgical revascularization for treatment of chronic mesenteric ischemia secondary to radiotherapy}

Background: Chronic mesenteric ischemia is observed in older people and is often due to atherosclerosis. Radiotherapy is an important risk factor for atherosclerosis. Aim: To report our experience with chronic mesenteric ischemia secondary to retroperitoneal radiotherapy. Material and Methods: Review of medical records of four male patients aged 39 to 65 years, treated for chronic mesenteric ischemia secondary to para-aortic radiotherapy between 1993 and 2011. Results: All of them had the classic symptoms of ischemia characterized by post prandial abdominal pain and weight loss. One had also isolated diarrhea episodes. Revascularization was achieved with open or endovascular surgery, with good results during a follow up period ranging from two to 20 years. Conclusions: Surgical revascularization is a good treatment for chronic mesenteric ischemia secondary to radiotherapy
\end{abstract}

Key words: Mesenteric ischemia, surgery, radiotherapy.

\section{Resumen}

Introducción: La insuficiencia mesentérica crónica es un diagnóstico infrecuente, generalmente secundario a enfermedad aterosclerótica, siendo considerada una enfermedad de pacientes añosos. Se sabe que la radioterapia es un factor de riesgo importante para aterosclerosis. Describimos nuestra experiencia en el manejo de la isquemia mesentérica crónica secundaria a radioterapia retroperitoneal. Materiales y Métodos: Análisis retrospectivo de las fichas clínicas de los pacientes tratados en nuestro centro por insuficiencia mesentérica crónica posterior a radioterapia del territorio para-aórtico entre 1993 y 2011. Resultados: Un total de 4 pacientes de sexo masculino fueron identificados. Edad promedio: $49 \pm 12$ años (rango 39-65). Todos presentaron los síntomas clásicos de insuficiencia mesentérica caracterizados por dolor abdominal postprandial y baja de peso. Uno de ellos además tenía episodios repetidos de diarrea. La revascularización mesentérica se obtuvo mediante cirugía abierta o endovascular con excelentes resultados a corto y largo plazo con un seguimiento promedio de 9,3 años (rango 2-20). Discusión: El curso acelerado de la aterosclerosis

*Recibido el 5 de marzo de 2015 y aceptado para publicación el 27 de marzo de 2015.

Autor no refiere conflictos de interés.

Correspondencia: Dr. José Francisco Vargas S.

jfvargas@gmail.com 
posterior a radioterapia se ha descrito en múltiples territorios vasculares. Síntomas de insuficiencia mesentérica crónica pueden ser malinterpretados en estos pacientes debido a su similitud con los síntomas observados en la ileitis actínica. Un diagnóstico y tratamiento oportuno son críticos para evitar complicaciones mayores y deterioro de calidad de vida de estos pacientes. Conclusión: La insuficiencia mesentérica crónica inducida por radioterapia es una condición infrecuente. El manejo con cirugía abierta o endovascular son seguras y otorgan resolución sintomática a largo plazo.

Palabras clave: Insuficiencia mesentérica crónica, isquemia mesentérica crónica, radioterapia retroperitoneal, radioterapia para-aórtica.

\section{Introducción}

El dolor abdominal postprandial recurrente asociado a pérdida progresiva de peso son los síntomas clásicos de la angina mesentérica. Dunphy acuñó el término en 1936 cuando describió doce pacientes que fallecieron por infartos intestinales. Todos ellos tuvieron una historia previa de angina intestinal que se había manifestado hasta varios años antes del desenlace fatal ${ }^{1}$.

Existen muchas causas de angina mesentérica pero la más frecuente es la aterosclerosis de las arterias mesentéricas; normalmente asociada a compromiso ateromatoso de otros territorios. $\mathrm{Su}$ incidencia aumenta con la edad y se estima que aproximadamente un $20 \%$ de la población europea y norteamericana sobre los 65 años está afectada ${ }^{2}$. Generalmente la progresión lenta del proceso estenótico de una única arteria visceral da tiempo para el desarrollo de múltiples vasos colaterales, lo que permite mantener una perfusión intestinal adecuada. Esto hace muy difícil lograr estimar la prevalencia real de la isquemia mesentérica ya que sus síntomas y la severidad de éstos serán muy variables ${ }^{3}$.

Los factores de riesgo principales para aterosclerosis son el tabaquismo, dislipidemia, hipertensión y diabletes mellitus ${ }^{3}$. Otro factor menos conocido, para un pequeño número de pacientes, es la radioterapia. Se sabe que la radiación genera daño arterial, pero hay escasos reportes de isquemia mesentérica crónica sintomática inducida por radioterapia ${ }^{4-10}$.

Reportamos cuatro pacientes tratados con radioterapia para-aórtica por diferentes cánceres en el pasado y que desarrollaron aterosclerosis acelerada del territorio mesentérico, con clínica evidente de insuficiencia mesentérica.

Se obtuvo aprobación del comité de ética institucional para la revisión de las fichas clínicas de estos pacientes.

\section{Caso 1}

Hombre de 41 años de edad que se trató con radioterapia para-aórtica 20 años antes de su presentación; dosis desconocidas.
Refería historia de dos años de evolución de dolor abdominal postprandial asociado a baja de peso no cuantificada. Angiografía demostró estenosis crítica del tronco celíaco y de la arteria mesentérica superior (AMS). Se confeccionaron sendos bypass aorto celíaco anterógrado y aorto mesentérico retrógrado con vena safena en otra institución.

Dos años después, en 1993, reapareció el dolor abdominal seguido de una baja de peso de 20 kilos. También refería claudicación intermitente de su extremidad inferior derecha. Angiografía demostró estenosis severa de su bypass aorto mesentérico y de la arteria iliaca común y externa derecha.

Se confeccionó un bypass aorto iliaco con prótesis de Dacrón y revascularización retrógrada de la arteria mesentérica superior con PTFE anillado (Figuras 1A y 1B).

Seis meses después el paciente ya había recuperado $20 \mathrm{~kg}$ y se encontraba asintomático. El paciente continúa asintomático 20 años después de su último procedimiento y tomografía axial computada en fase arterial demostró permeabilidad de todas las reconstrucciones (Figura 2).

\section{Caso 2}

Paciente de 39 años de edad tratado por cáncer testicular con orquidectomía, linfadenectomía lumboaórtica, quimio y radioterapia 18 años antes de su presentación. Recibió 4.600 cGy al campo inguino ilíaco y 4.600 cGy a la región lumboaórtica (180 cGy diarios por 5 semanas).

En 1999, diecisiete años luego de haber completado su radioterapia, se presentó con claudicación intermitente invalidante y dolor abdominal postprandial con discreta disminución de peso y diarrea.

Estudio no invasivo fue compatible con oclusión aortoilíaca que se confirmó con angiografía, la que además demostró estenosis crítica de la AMS a 2 $\mathrm{cm}$ del ostium. Se realizó angioplastía con balón de esta lesión y revascularización extraanatómica de sus extremidades inferiores mediante bypass áxilobifemoral, siendo dado de alta al séptimo día postoperatorio con resolución completa de sus síntomas.

Seis meses después se presentó con re-estenosis 


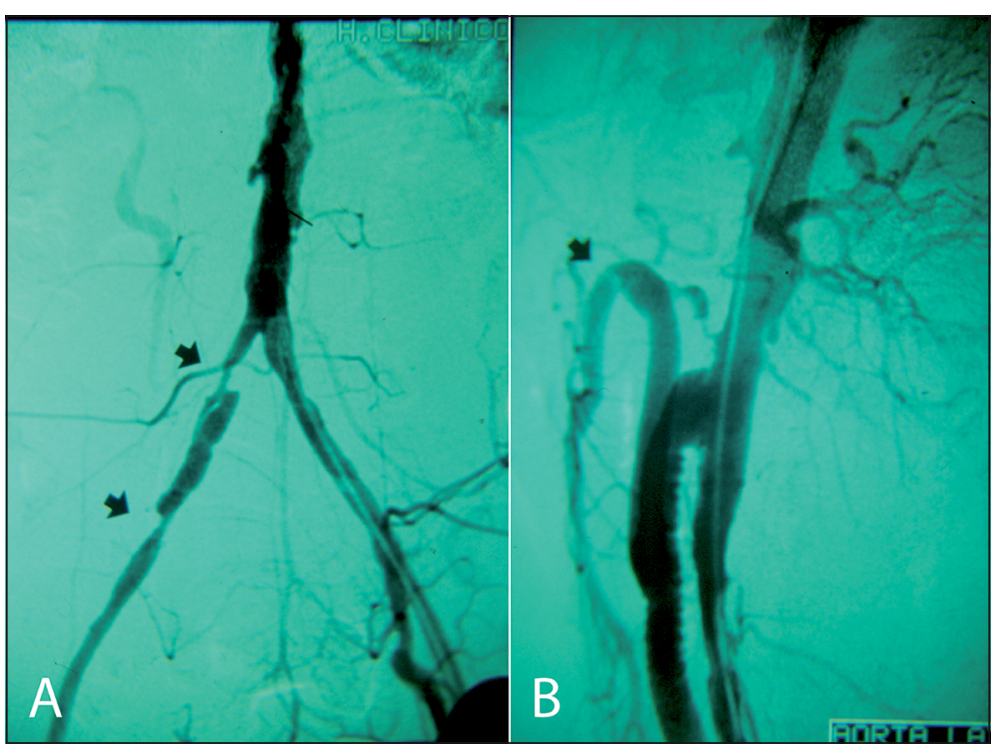

Figura 1. A) Angiografía preoperatoria que muestra oclusiones viscerales y estenosis ilíaca; B) Angiografía postoperatoria tomada luego de los bypass aorto ilíaco e ilio mesentérico. clínica y angiográfica de su AMS. Angioplastía con stent se completó sin incidentes. (Cordis SMART ${ }^{\circledR}$ 7 x 40 mm; Cordis Corp, Miami, FL).

Un año después se presentó con hipertensión severa y estudio con ecografía dúplex arterial

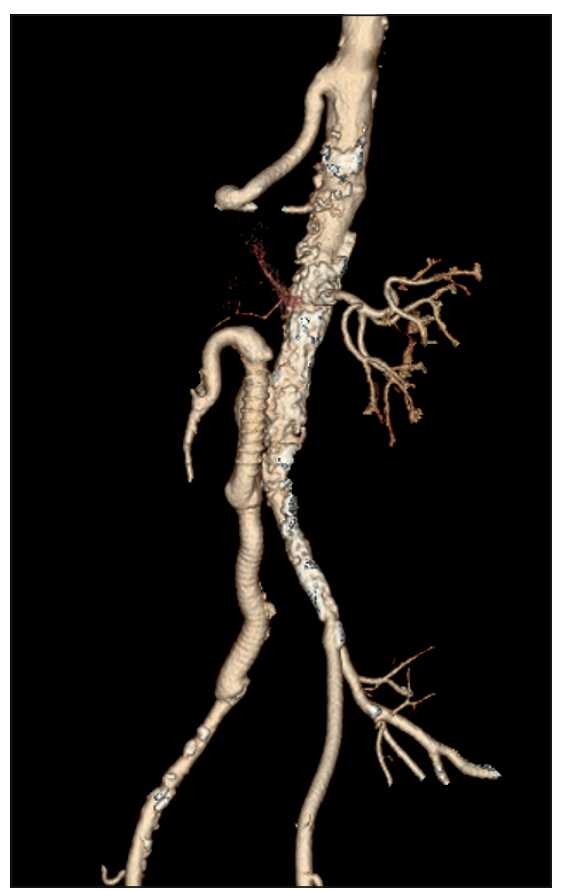

Figura 2. Tomografía computada en fase arterial tomada 20 años después de la última cirugía del paciente. demostró una estenosis crítica de la arteria renal derecha que fue tratada mediante angioplastía con balón. Angiografía durante ese procedimiento demostró permeabilidad de su stent mesentérico sin re-estenosis significativa.

Desarrolló falla renal progresiva por fibrosis retroperitoneal y obstrucción ureteral iniciando hemodiálisis 8 años después. En ese momento estudio con tomografía computada en fase arterial demostró permeabilidad de ambas arterias renales y del stent mesentérico.

Once años después de su angioplastía con stent mesentérico requirió cirugía por cáncer de colon transverso. Antes de esa cirugía se completó estudio con angiografía selectiva de la AMS demostrándose estenosis asintomática de $70 \%$ proximal al dispositivo, que fue tratada con nuevo stent (Cordis PRECISE $^{\circledR} 8 \times 40 \mathrm{~mm}$; Cordis Corp, Miami, FL) (Figura 3). Fue operado con éxito de su cáncer de colon y ha estado libre de eventos y de síntomas de insuficiencia mesentérica durante los últimos dos años; 13 años después de su primera angioplastía mesentérica con stent.

\section{Caso 3}

Hombre de 65 años con antecedentes de tabaquismo recientemente suspendido (25 paquetes/ año). Fue tratado por cáncer testicular con cirugía y radioterapia (dosis desconocida) 30 años antes de su consulta.

Se presentó en el año 2011 con una historia de 2 


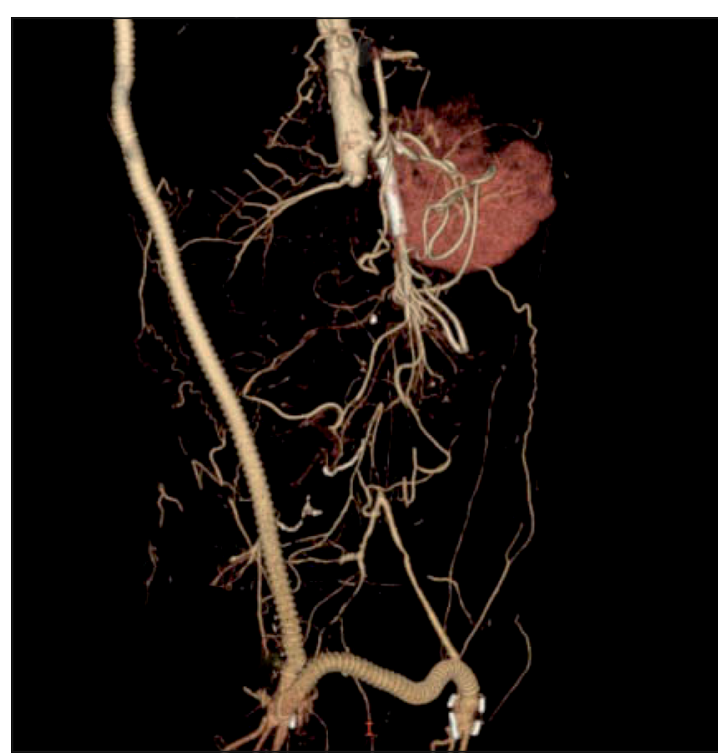

Figura 3. Tomografía computada en fase arterial que muestra permeabilidad del bypass axilo bifemoral y del stent mesentérico.

años de evolución de dolor abdominal epigástrico asociado a baja de peso de 20 kilos en ese período.

Tomografía computada demostró ateromatosis aórtica severa restringida al área previamente irradiada con posible oclusión del origen de la AMS. Tronco celíaco no se pudo visualizar con claridad. (Figura 4A).

Angiografía a través de acceso braquial confirmó estenosis de $50 \%$ del origen del tronco celíaco y una oclusión del segmento proximal de la AMS de $3 \mathrm{~cm}$ de largo. No se intentó recanalizar esta lesión por su extensa calcificación. Se confeccionó un by pass ilio mesentérico con prótesis de Dacrón de $8 \mathrm{~mm}$ (Figura 4B).

Fue dado de alta dos semanas después de su cirugía aumentando su ingesta progresivamente. A 2 años está libre de síntomas y controles con ecografía dúplex han demostrado permeabilidad del bypass.

\section{Caso 4}

Paciente de 50 años de edad sometido a tratamiento combinado con quimio-radioterapia de linfoma de Hodgkin 25 años antes de su presentación; dosis desconocidas. Presentaba múltiples secuelas secundarias a su radioterapia: pericarditis constrictiva y estenosis sintomática difusa de ambas carótidas manejada médicamente.

Al ser evaluado en el año 2011 refería historia de 15 años de evolución de dolor abdominal postprandial lentamente progresivo con pérdida de peso importante que fue interpretada por su oncólogo y múltiples gastroenterólogos como ileitis actínica.

Tomografía axial computada en fase arterial evidenció estenosis significativa de los orígenes de la AMS y del tronco celíaco (Figura 5). Angiografía confirmó estenosis de $70 \%$ del tronco celíaco y $90 \%$ del origen de la AMS.

Se completó tratamiento con angioplastía e implante de stent a ambos vasos viscerales: (SMARTÒ 7x30 mm al tronco celíaco y otro de $8 \times 40 \mathrm{~mm}$ SMARTÒ a la AMS; Cordis Corp, Miami, FL). Se toleró estenosis residual de $50 \%$ del tronco celíaco (Figura 6). Fue dado de alta el segundo día postoperatorio y permanece asintomático a los dos años.
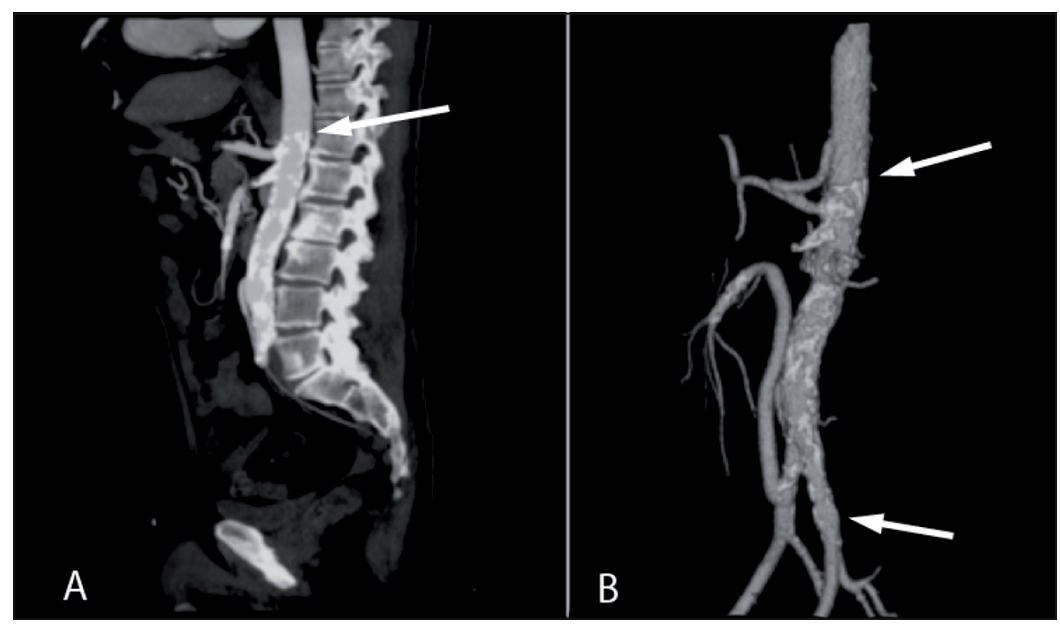

Figura 4. A) Tomografía computada en fase arterial preoperatoria que muestra las calcificaciones extensas del territorio irradiado con estenosis moderada del tronco celíaco y gruesas calcificaciones de la arteria mesentérica superior; B) Tomografía computada en fase arterial postoperatoria que muestra el bypass ilio mesentérico. Notar la clara delimitación entre el territorio sano y el afectado por la radiación (flechas). 


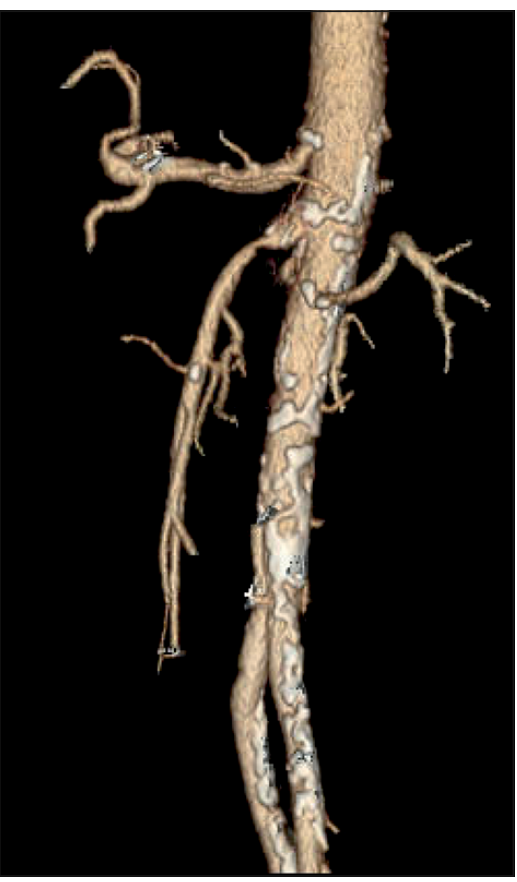

Figura 5. Tomografía computada en fase arterial que muestra estenosis del origen del tronco celíaco y la arteria mesentérica superior.

\section{Discusión}

Se piensa que hasta $60 \%$ de los pacientes con cáncer requerirán radioterapia en algún momento de su tratamiento ${ }^{8}$. Las complicaciones vasculares del territorio tratado son difíciles de predecir pero sus consecuencias deben ser comprendidas y diagnosticadas oportunamente.

Al final del siglo XIX Gassman demostró la existencia de daño a lechos arteriales por radioterapia, las consecuencias del mismo a nivel cardiaco y cervical fueron demostradas durante el siglo pasado ${ }^{4,8,9}$. Los pacientes que requieren radioterapia cervical pueden tener una incidencia de estenosis carotídea secundaria de hasta $25 \%$ y aproximadamente $12 \%$ de ellos desarrollarán síntomas.

La inflamación generada por la radioterapia produce daño endotelial y activa la cascada de coagulación llevando a deposición de fibrina9. Hay transformación acelerada de fibroblastos a fibrocitos y perpetuación de la injuria por la liberación de radicales libres. Todos estos cambios precipitarán el desarrollo del proceso aterosclerótico normal en los vasos sometidos a radioterapia ${ }^{4,8,9}$.

El daño arterial inducido por radioterapia ha sido descrito en todas las arterias principales incluyendo la aorta, iliacas, femorales, coronarias, subclavias,

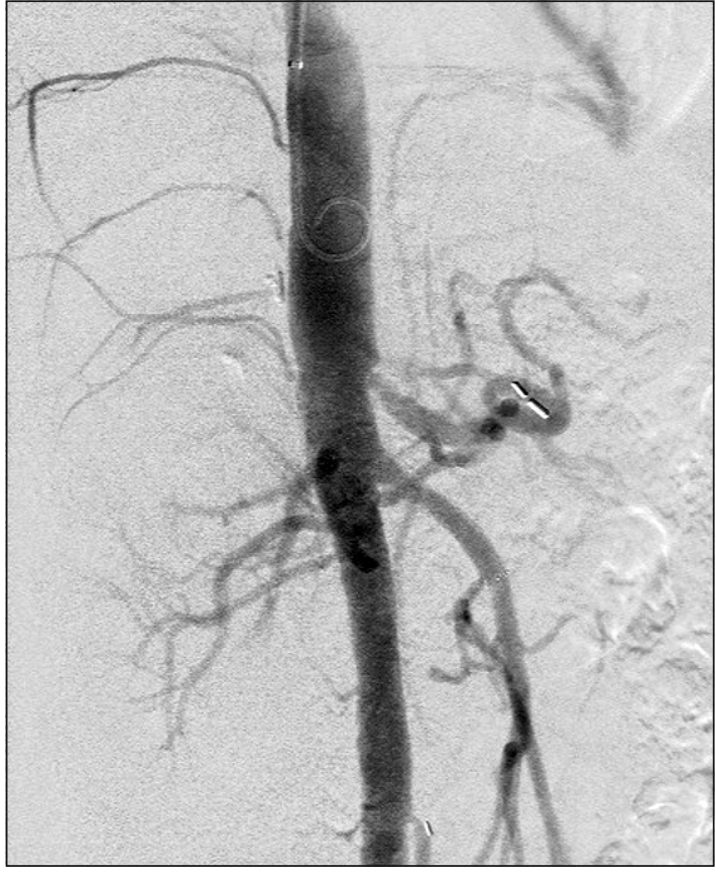

Figura 6. Angiografía de control luego de angioplastía con stent del tronco celíaco y de la arteria mesentérica superior.

braquiales y carótidas extracraneanas ${ }^{8,9,11,12}$. No hay estudios prospectivos que analicen la incidencia de este daño en el territorio mesentérico pero hay descripciones de casos que destacan su importancia clínica. Es así como Israeli et al. ${ }^{13}$, describieron dos pacientes que habían sido sometidos a radioterapia pelviana y sufrieron isquemia colónica aguda y severa luego de la reparación de aneurismas infrarrenales.

La incidencia de daño arterial es mayor en aquellos pacientes que además presentan otros factores de riesgo de aterosclerosis, siendo proporcional a la dosis de radioterapia recibida; una dosis única de 1.500 cGy o múltiples dosis que sumadas sean mayores a 3.000-4.000 cGy desarrollarán daño arterial $1^{14,15}$. El riesgo cardiovascular normal se elevará 2-7 veces luego de recibir dosis acumuladas de 3.000 a 4.000 cGy, pero incluso hay casos claramente documentados de daño coronario con dosis menores a 160 a $390 \mathrm{cGy}^{14-16}$. Estos podrían deberse a una mayor elevación de citoquinas, disminución de la inmunidad de células $\mathrm{T} u$ otros factores en esos pacientes ${ }^{15}$. Estudios experimentales en animales han confirmado que la asociación de radioterapia a una dieta rica en colesterol e hipertensión elevan la incidencia de daño en el territorio irradiado significativamente ${ }^{17}$. A pesar de que sólo fue posible ob- 
tener la dosis de irradiación de uno de los pacientes podemos asumir por los protocolos de tratamiento usados en esa época que los otros probablemente recibieron alrededor de $4.000 \mathrm{cGy}$.

Es importante recalcar que este proceso de aterosclerosis acelerada debe diferenciarse de otras causas de isquemia mesentérica (aterosclerosis no inducida por radioterapia, vasculitis, síndrome de ligamento arcuato, síndrome de aorta media y otros)

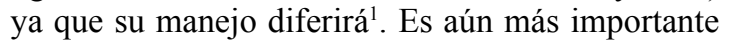
diferenciarla de aquellos casos de enteritis actínica ya que sus síntomas serán similares y frecuentemente coexistentes.

El daño al territorio mesentérico generado por la radioterapia se ha descrito luego del tratamiento de múltiples cánceres, principalmente testiculares y linfáticos como nuestros pacientes. Las manifestaciones son variables y van desde la oclusión completamente asintomática hasta la trombosis aguda o, menos frecuentemente, la rotura ${ }^{9}$. Es importante enfatizar que el daño a estructuras vecinas complica el desarrollo de redes colaterales adecuadas, lo que lleva a la presentación de síntomas. Se cree que la rotura ocurre cuando hay además infección y destrucción secundaria de la pared arterial dañada ${ }^{18}$.

Un tercio de los pacientes presentarán síntomas en los primeros 5 años luego de su tratamiento y otro tercio se mantendrá asintomático por sobre la primera década ${ }^{10}$. Todos los médicos involucrados en el tratamiento y seguimiento de los pacientes irradiados deben estar alerta ante síntomas posibles de isquemia de los territorios irradiados (Figuras 2, 4 y 5). Este riesgo se mantiene en el tiempo y medidas simples como la historia clínica y un examen físico prolijo pueden asistir para un diagnóstico adecuado y oportuno. Todos nuestros pacientes comenzaron con síntomas al menos 15 años después de haber completado su tratamiento por cáncer. Estuvieron con síntomas por más de un año y consultaron a múltiples especialistas buscando respuesta a sus molestias. La juventud de al menos 3 de ellos hizo que este diagnóstico fuera aún más difícil.

Una vez que se ha detectado la presencia de aterosclerosis sintomática en pacientes previamente sometidos a radioterapia, el manejo inicial será idéntico al del paciente no irradiado. A pesar de la evidencia escasa, se debe controlar cada factor de riesgo conocido. Los objetivos iniciales serán la normalización de la presión arterial, corrección de la dislipidemia y suspensión del tabaco. Se promueve el uso precoz de estatinas, por su efecto antiinflamatorio a nivel de la placa aterosclerótica; y algunos autores señalan que debe instaurarse incluso profilácticamente?

El estudio diagnóstico inicial puede involucrar cualquier modalidad no invasiva: ecografía dúplex, tomografía computada o resonancia nuclear magnética. La angiografía se reserva para confirmar un diagnóstico que aún no se ha aclarado con los estudios ya mencionados o para planificar o ejecutar el tratamiento ${ }^{19}$.

Existe una tendencia global a tratar la mayoría de los pacientes con insuficiencia mesentérica de origen aterosclerótico por vía endovascular. A pesar de sus mejores tasas de permeabilidad primaria y secundaria, la cirugía convencional abierta se reserva para pacientes con alguna contraindicacón para el manejo endovascular ${ }^{1,19-21}$. Las tasas de reintervención de los procedimientos endovasculares se han descrito que alcanzan hasta 3 a 4 veces aquellos de la cirugía abierta pero, usando este manejo inicial, las tasas de mortalidad se han reducido desde 3,4$15 \%$ hasta $1,7-4 \%{ }^{21-24}$. Además, las reintervenciones pueden resolverse vía endovascular la mayoría de las veces ${ }^{25}$.

Tuvimos muy buenos resultados a corto y largo plazo en los 4 pacientes salvo la reestenosis precoz del paciente tratado con angioplastía primaria con balón en el año 1999, seguido de una angioplastía con stent que fue exitosa a largo plazo. Actualmente ese paciente sería tratado así desde un principio, basándose principalmente en la experiencia obtenida de los estudios de estenosis carotídea post-actínica $\mathrm{y}$ aterosclerosis regular de vasos viscerales. Todos nuestros casos fueron tratados en los últimos 30 años y cada uno se enfrentó con la evidencia y opciones de tratamiento disponibles para la época en que se presentaron.

Los cambios inducidos por la radioterapia no sólo afectan las estructuras vasculares, sino todas las estructuras que rodean los vasos afectados, por lo que la cirugía abierta se hace aún más demandante por la fibrosis perivascular. En nuestros pacientes tuvimos excelentes resultados perioperatorios y a largo plazo con las técnicas abiertas y endovasculares. Esto ya ha sido descrito en otros centros con alto volumen de cirugía vascular que reportan tasas bajas de morbimortalidad a pesar de haber reducido la proporción de casos abiertos vs endovasculares ${ }^{23}$.

\section{Conclusión}

La isquemia mesentérica crónica inducida por radioterapia es un diagnóstico infrecuente que debe ser descartado en un grupo selecto de pacientes. $\mathrm{Su}$ similitud clínica, con la enteritis actínica que es sumamente más frecuente, hace que el diagnóstico sea rara vez buscado por los oncólogos o gastroenterólogos que los tratan. Una vez confirmado, el tratamiento endovascular o abierto ofrece excelentes resultados a corto y largo plazo. 


\section{Referencias}

1. Oderich GS. Current concepts in the management of chronic mesenteric ischemia. Curr Treat Options Cardiovasc Med. 2010;12:117-30.

2. Acosta S. Epidemiology of mesenteric vascular disease: clinical implications. Semin Vasc Surg. 2010;23:4-8.

3. Huerta C, Rivero E, Montoro MA, García-Rodríguez LA. Risk factors for intestinal ischaemia among patients registered in a UK primary care database: a nested casecontrol study. Aliment Pharmacol Ther. 2011;33:969-78.

4. Pherwani AD, Reid JA, Keane PF, Hannon RJ, Soong $\mathrm{CV}$, Lee B. Synergism between radiotherapy and vascular risk factors in the accelerated development of atherosclerosis: a report of three cases. Ann Vasc Surg. 2002; 16:671-5.

5. Atabek U, Spence RK, Alexander JB, Pello MJ, Camishion RC. Upper extremity occlusive arterial disease after radiotherapy for breast cancer. J Surg Oncol. 1992;49:205-7.

6. Chun CL, Joye J, Triadafilopoulos G. Holiday Pains: A Case of Radiation-Induced Mesenteric Ischemia. Dig Dis Sci. 2012;58:349-53

7. Tomori H, Yasuda T, Shiraishi M, Isa T, Muto Y, Egawa H. Radiation-associated ischemic coloproctitis: report of two cases. Surg Today. 1999;29:1088-92.

8. Katras T, Baltazar U, Colvett K, Rush D, Dunn J, Stanton P, Jr. Radiation-related arterial disease. Am Surg. 1999;65:1176-9.

9. Jurado JA, Bashir R, Burket MW. Radiation-induced peripheral artery disease. Catheter Cardiovasc Interv. 2008;72:563-8.

10. Himmel PD, Hassett JM. Radiation-induced chronic arterial injury. Semin Surg Oncol. 1986;2:225-47.

11. Budin JA, Casarella WJ, Harisiadis L. Subclavian artery occlusion following radiotherapy for carcinoma of the breast. Radiology 1976;118:169-73.

12. McCready RA, Hyde GL, Bivins BA, Mattingly SS, Griffen WO Jr. Radiation-induced arterial injuries. Surgery 1983;93:306-12.

13. Israeli D, Dardik H, Wolodiger F, Silvestri F, Scherl B, Chessler R. Pelvic radiation therapy as a risk factor for ischemic colitis complicating abdominal aortic reconstruction. J Vasc Surg. 1996;23:706-9.

14. Bhatti P, Sigurdson A J, Mabuchi K. Can low-dose ra- diation increase risk of cardiovascular disease? Lancet 2008;372:697-9.

15. Stewart FA. Mechanisms and dose-response relationships for radiation-induced cardiovascular disease. Ann ICRP 2012;41:72-9.

16. Carr ZA, Land CE, Kleinerman RA, Weinstock RW, Stovall M, Griem ML, et al. Coronary heart disease after radiotherapy for peptic ulcer disease. Int J Radiat Oncol Biol Phys. 2005;61:842-50.

17. Gold H. Production of arteriosclerosis in the rat. Effect of x-ray and a high-fat diet. Arch Pathol. 1961;71:26873.

18. Marcial-Rojas RA, Castro JR. Irradiation injury to elastic arteries in the course of treatment for neoplastic disease. Ann Otol Rhinol Laryngol. 1962;71:945-58.

19. Pecoraro F, Rancic Z, Lachat M, Mayer D, Amann-Vesti B, Pfammatter T, et al. Chronic Mesenteric Ischemia: Critical Review and Guidelines for Management. Ann Vasc Surg. 2012;27:113-22.

20. Gupta PK, Horan SM, Turaga KK, Miller WJ, Pipinos II. Chronic mesenteric ischemia: endovascular versus open revascularization. J Endovasc Ther. 2010;17:5409.

21. Oderich GS, Gloviczki P, Bower TC. Open surgical treatment for chronic mesenteric ischemia in the endovascular era: when it is necessary and what is the preferred technique? Semin Vasc Surg. 2010;23:36-46.

22. Oderich GS, Tallarita T, Gloviczki P, Duncan AA, Kalra $\mathrm{M}$, Misra S, et al. Mesenteric artery complications during angioplasty and stent placement for atherosclerotic chronic mesenteric ischemia. J Vasc Surg. 2012;55:1063-71.

23. Ryer EJ, Oderich GS, Bower TC, Macedo TA, Vrtiska TJ, Duncan AA, et al. Differences in anatomy and outcomes in patients treated with open mesenteric revascularization before and after the endovascular era. J Vasc Surg. 2011;53:1611-8 e1612.

24. Tallarita T, Oderich GS, Macedo TA, Gloviczki P, Misra $\mathrm{S}$, Duncan AA, et al. Reinterventions for stent restenosis in patients treated for atherosclerotic mesenteric artery disease. J Vasc Surg. 2011;54:1422-9 e1421.

25. Sarac TP, Altinel O, Kashyap V, Bena J, Lyden S, Sruvastava S, et al. Endovascular treatment of stenotic and occluded visceral arteries for chronic mesenteric ischemia. J Vasc Surg. 2008;47:485-91. 